\title{
Acupuncture in Temporomandibular Disorders Painful Symptomatology: An Evidence-Based Case Report
}

\author{
Lia Dietrich ${ }^{1,2}$ Igor Vinícius Santos Rodrigues ${ }^{1}$ Marcelo Dias Moreira de Assis Costa ${ }^{3,4}$ \\ Roberta Furtado Carvalho ${ }^{5}$ Gisele Rodrigues da Silva ${ }^{1}$
}

${ }^{1}$ Department of Operative Dentistry and Dental Materials, Biomechanics Research Group, School of Dentistry, Federal University of Uberlândia, Uberlândia, Brazil

${ }^{2}$ Department of Operative Dentistry and Oral Rehabilitation, Faculty of Dentistry, Faculty of Patos de Minas, Patos de Minas, Brazil

3 Integrated Dental Clinic Program, Faculty of Dentistry, Federal University of Uberlândia, Minas Gerais, Brazil

${ }^{4}$ Department of Oral Surgery, Faculty of Dentistry, Faculty of Patos de Minas, Patos de Minas, Brazil

${ }^{5}$ Department of Operative Dentistry and Dental Materials, Biomechanics Research Group, School of Dentistry, CEUMA University/Federal University of Uberlândia, Uberlândia, Brazil
Address for correspondence Gisele Rodrigues da Silva, DDS, MSc, PhD, Department of Operative Dentistry and Dental Materials, Dental School, Federal University of Uberlândia, Piratini Republic Avenue, s/ $\mathrm{n}^{\circ}$, 4L Block, Umuarama 38400902 Uberlândia, MG, Brazil (e-mail: giselerosilva@yahoo.com.br).

\author{
Abstract \\ Keywords \\ - acupuncture \\ - case report \\ - tempormandibular \\ dysfunction \\ - traditional Chinese \\ medicine
}

Temporomandibular dysfunction (TMD) is complex and multifactorial. Its etiology involves various factors, such as malocclusion, psychological patterns, parafunctional habits, and the temporomandibular joint anatomy. Symptoms include myofascial pain, joint clicking, mouth opening limitation, headaches, earaches, and neck pain, comprising one of the greatest causes of nondental pain. Acupuncture has been used to treat these conditions by acting locally both in muscle relaxation and pain management as well as reaching physical, mental, and emotional balance. The aim of this study is to present a brief literature review reporting the efficacy of acupuncture on TMDs and a case report in which the painful symptomatology of TMD was treated with a systemic protocol, based on scientific evidence and concepts of traditional Chinese medicine. Acupuncture may be a complementary treatment for TMD, and in this case report, it eliminated the patient's painful symptomatology.

\section{Introduction}

Temporomandibular dysfunction (TMD) refers to different kinds of conditions that affect the temporomandibular joints (TMJs), masticatory muscles, and associated structures of the head and neck. ${ }^{1}$ Signs and symptoms linked to TMD are debilitating pain in the orofacial region, TMJ pain and/or clicking sounds, mouth opening limitation, restriction in mandibular movements, and presence of trigger points (TPs) on palpation. ${ }^{2-5}$ TMD consists a major cause of nondental pain, more frequent in people from 20 to 40 years, and twice as common in females than in males. ${ }^{6,7}$

Its etiology is multifactorial, involving several factors such as parafunctional habits (bruxism, teeth clenching), trauma to the jaw and surrounding areas, degenerative joint diseases, postural changes, TMJ anatomy, hormonal alterations, and maxillomandibular relationship instability. ${ }^{1}$ Studies show that emotional factors (stress, anxiety, depression) also play an important role in TMDs. ${ }^{1,9,9}$

Due to TMDs' complex characteristics, conservative treatment modalities include education, self-care, neuromyorelaxing occlusal splints, pharmacotherapy, and behavioral therapy. Relaxation techniques and acupuncture have been used as complementary therapy in patients with TMD painful symptomatology. ${ }^{1,2,4}$

Acupuncture originated in China over 3,000 years ago consisting one of the building blocks of traditional Chinese medicine (TCM) and it has been used to treat plenty clinical conditions, especially in pain management. ${ }^{23,10}$ Since pain is 
often observed in patients with TMD, acupuncture has been widely chosen as adjunctive therapy, with a considerable number of studies corroborating to its effectiveness., ${ }^{2,4,11,12}$

The aim of this case report is to present a case report of a patient diagnosed with TMD, submitted to an acupuncture protocol treatment based on scientific evidences and TCM concepts, offering a more individualized approach.

\section{Case Report}

This clinical case is part of a research examination approved by the Patos de Minas School of Dentistry Committee for Research Involving Human Subjects (number approved 2.439.313 and CAAE: 75474217.1.0000.8078).

The inclusion criteria of the patient in this study were the presence of pain by TMD of muscular origin or mixed, with or without mouth opening limitation, according to the research diagnostic criteria for temporomandibular disorders (RDC/ TMD), Angle's Class I, and no absence of teeth up to second molar.

A 22-year-old woman complaining of severe pain in the middle third of the face radiating to the head searched for treatment. Patient had no systemic alterations and reported that pain begun 2 months prior to the first appointment and was managing it by taking analgesic (dipyrone $500 \mathrm{mg}$ ) and anti-inflammatory (ibuprofen $400 \mathrm{mg}$ ) on her own. However, pain became worse and medication was not being effective anymore.

At clinical examination, patient reported pain score 10 in the $10 \mathrm{~cm}$ visual analog scale (VAS), where 0 represents "no pain" and 10 the "worst pain possible." Occlusion evaluation and muscle palpation were performed in accordance with the RDC/TMD (Axis I). ${ }^{13}$

The patient had premature contacts on the anterior teeth and occlusal interference on right lateral movement (LR). Intense pain was reported in the masseter, temporalis, trapezius, and sternocleidomastoid muscles bilaterally during palpation examination, as well as mouth opening limitation, pain on mastication, especially food of harder consistency. Patient also reported being under stress because of daily routine of studies and a night shift job. The RDC/TMD (Axis II) ${ }^{13}$ questionnaire was applied to confirm TMD diagnosis. According to TMD (RDC/TMD), the patient was diagnosed with muscle disorders with myofascial pain and limited mouth opening, where the pain was directly related to the masticatory and cervical muscles. Five acupuncture sessions (one session a week-10 minutes) were proposed to the patient to minimize painful symptomatology. The utilized points were: ST6, ST7, SI19, TE21, Yintang, TPs, GV20, LI4, SI3, GB34, GB20, and ST36. To make muscle activity assessment before and after treatment, the patient underwent electromyography (EMG) test. ${ }^{14}$ The handler used the EMG030204/06U (EMG System do Brasil Ltda) equipment with four channels, 14 bits resolution in signal acquisition, and 5,000-volt electrical isolation. The software (EMG System do Brasil-www.emgsystem. com.br) was used for assessment.

The skin was cleaned with cotton wool and 70\% alcohol before signal acquisition. Disposable bipolar $\mathrm{Ag} / \mathrm{AgCl}$ electrodes of $1 \mathrm{~cm}$, which were circular in shape, were connected to the preamplifier and placed at a distance of $2 \mathrm{~cm}$ from each other according to Surface ElectroMyoGraphy for Non-invasive Assessment of Muscles (SENIAM) Project ${ }^{15}$ on the following muscles: first the masseter and then the temporalis (anterior portion), bilaterally.

The electrical activity of these muscles was recorded at rest $(\mathrm{R})$, maximum voluntary contraction, maximum protrusion (P), LR, left lateral movement, clenching wax, habitual mastication, chewing peanut, and chewing raisin. EMG test was performed right before the first acupuncture session (T0), right after it (T1), and after the fifth (T2) session (-Table 1). During the test, patient remained sitting in a comfortable position, and was oriented to keep the back straight, arms relaxed, feet on the floor, and eyes fixed on the horizon. Patient was advised to interrupt self-medication 1 week prior to the first session. At week 1 , VAS score was 8 before EMG test and 10 after it (T0). VAS score was 0 after the first acupuncture session and 3 after EMG test (T1). As the patient reported greater discomfort on the left side of the face on that day, only acupoints on the left side of the body were punctured. On the following sessions, acupoints of the both sides were selected for puncturing. After the last session, the patient reported no more painful symptoms.

The acupoints selected for this case were those recommended based on TCM, by the literature, for orofacial pain ${ }^{2,3}$ as well as TPs (Ashe) $)^{16}$ and points for anxiety (-Table 2). Also was considered the Ashi point which is an unconventional point on the individual's body that is sensitive to touch, and may be present on the integument or muscle, being identified through digital pressure, next to the main complaint region. Punctures with 5 to $15 \mathrm{~mm}$ depth, appropriate to the targeted muscle or point were performed with stainless steel acupuncture needles (DongBang; DBC 108 Medical Co Ltd, South Korea) were chosen in sizes $0.22 \mathrm{~mm} \times 13 \mathrm{~mm}$ (for points of the face) and $0.25 \mathrm{~mm} \times 30 \mathrm{~mm}$ (for distal points). All inserted and stimulated manually for 10 minutes, bilaterally. For the precise puncturing of active TPs, palpation and localization of taut muscle bands and TPs on the most important masticatory and cervical muscles were performed. The needles were inserted in these spots (5-15 mm depth). The skin was previously cleaned on the point's location with cotton wool and $70 \%$ alcohol. During therapy, the patient stayed lying on a stretcher in supine position.

The patient underwent occlusal adjustment after the first acupuncture session and will receive a myorelaxing occlusal splint to protect the teeth and neuromuscular system from parafunctional activities such as teeth clenching and bruxism. ${ }^{1}$

\section{Discussion}

TMDs are multifactorial disorders, which appear as painful symptomatologies and/or functional alterations of the mandible, needed to be investigated through patient's historic, clinical, and psychobehavioral evaluations, for a therapeutic planning directed to pain or dysfunction. ${ }^{3,5,17-19}$ It displays a predominance in individuals with age range of 20 to 40 years, 
Table 1 Electromyography test

\begin{tabular}{|c|c|c|c|c|c|c|c|c|c|c|c|}
\hline \multirow[t]{2}{*}{ Muscle } & \multirow[t]{2}{*}{ Data } & \multirow[t]{2}{*}{ Channel } & \multicolumn{9}{|c|}{ Muscular electrical activity } \\
\hline & & & $\mathrm{R}$ & MVC & $\mathbf{P}$ & LR & LL & $\mathrm{CW}$ & HM & $\mathrm{CP}$ & CR \\
\hline \multirow[t]{12}{*}{ Masseter } & \multirow[t]{6}{*}{ RMS } & C3-T0 & 56.6 & 66.51 & 61.92 & 54.48 & 58.36 & 77.41 & 66.63 & 63.48 & 64.55 \\
\hline & & $\mathrm{C} 3-\mathrm{T} 1$ & 48.44 & 50.48 & 42.77 & 45.94 & 48.43 & 68.14 & 52.6 & 64.8 & 64.89 \\
\hline & & $\mathrm{C} 3-\mathrm{T} 2$ & 40.94 & 35.15 & 41.98 & 45.05 & 44.09 & 66.06 & 51.33 & 63.47 & 55.54 \\
\hline & & C4-T0 & 52.03 & 53.37 & 52.92 & 50.1 & 36.67 & 66.77 & 69.12 & 59.84 & 59.84 \\
\hline & & $\mathrm{C} 4-\mathrm{T} 1$ & 19.48 & 27.53 & 32.95 & 24.83 & 20.85 & 49.42 & 39.18 & 45.38 & 39.57 \\
\hline & & C4-T2 & 16.15 & 26.41 & 30.42 & 28.23 & 25.30 & 48.00 & 38.89 & 37.02 & 38.37 \\
\hline & \multirow[t]{6}{*}{ Area } & C3-T0 & 44.53 & 23.45 & 47.19 & 46.23 & 46.81 & 48.87 & 48.85 & 48.96 & 48.96 \\
\hline & & C3-T1 & 51.01 & 51.01 & 48.77 & 50.22 & 50.24 & 51.68 & 50.17 & 51.15 & 51.25 \\
\hline & & $\mathrm{C} 3-\mathrm{T} 2$ & 44.96 & 22.67 & 45.32 & 44.87 & 44.46 & 46.78 & 44.17 & 44.58 & 44.10 \\
\hline & & C4-T0 & 18.8 & 10.11 & 20.37 & 20.15 & 19.19 & 22.84 & 22.56 & 22.56 & 22.65 \\
\hline & & $\mathrm{C} 4-\mathrm{T} 1$ & 22.37 & 22.37 & 22.15 & 21.39 & 22.36 & 23.69 & 22.59 & 22.81 & 22.03 \\
\hline & & $\mathrm{C} 4-\mathrm{T} 2$ & 14.82 & 10.14 & 20.38 & 19.87 & 19.09 & 22.55 & 20.02 & 20.57 & 20.76 \\
\hline \multirow[t]{12}{*}{ Temporalis } & \multirow[t]{6}{*}{ RMS } & C3-T0 & 27.37 & 104.88 & 17.24 & 18.09 & 16.14 & 138.95 & 89.04 & 76.48 & 71.62 \\
\hline & & $\mathrm{C} 3-\mathrm{T} 1$ & 19.02 & 109.08 & 16.67 & 17.69 & 17.6 & 168.72 & 75.57 & 99.75 & 88.2 \\
\hline & & $\mathrm{C} 3-\mathrm{T} 2$ & 18.15 & 92.98 & 15.62 & 17.39 & 16.49 & 136.91 & 60.32 & 80.09 & 69.05 \\
\hline & & C4-T0 & 29.86 & 65.34 & 31.3 & 28.25 & 40.13 & 156.05 & 112.09 & 113.64 & 91.75 \\
\hline & & $\mathrm{C} 4-\mathrm{T} 1$ & 39.58 & 71.71 & 26.23 & 26.37 & 26.85 & 147.55 & 67.21 & 102.59 & 86.61 \\
\hline & & C4-T2 & 36.72 & 70.95 & 22.48 & 26.55 & 24.52 & 144.56 & 50.99 & 101.18 & 86.68 \\
\hline & \multirow[t]{6}{*}{ Area } & C3-T0 & 43.94 & 25.53 & 42.99 & 43.02 & 43.11 & 52.3 & 46.78 & 45.89 & 46.69 \\
\hline & & $\mathrm{C} 3-\mathrm{T} 1$ & 43.57 & 25.71 & 44.09 & 44.01 & 44.15 & 54.18 & 46.15 & 46.8 & 46.29 \\
\hline & & $\mathrm{C} 3-\mathrm{T} 2$ & 41.86 & 24.18 & 41.99 & 42.62 & 42.77 & 50.38 & 46.03 & 43.92 & 43.14 \\
\hline & & C4-T0 & 22.44 & 11.76 & 22.13 & 21.65 & 22.71 & 28.14 & 26.29 & 25.5 & 24.77 \\
\hline & & $\mathrm{C} 4-\mathrm{T} 1$ & 21.72 & 12.96 & 21.48 & 21.24 & 21.4 & 28.42 & 22.23 & 24.1 & 23.31 \\
\hline & & $\mathrm{C} 4-\mathrm{T} 2$ & 20.36 & 11.47 & 17.96 & 17.19 & 18.08 & 25.56 & 18.00 & 21.26 & 19.62 \\
\hline
\end{tabular}

Abbreviations: $\mathrm{CP}$, chewing peanut; $\mathrm{CR}$, chewing raisin; CW, clenching wax; HM, habitual mastication; LL, left lateral movement; LR, right lateral movement; MVC, maximum voluntary contraction; P, protrusion; R, rest; RMS, root mean square.

feminine gender. ${ }^{6,711}$ The patient of this study fits this age range and gender, and the proposed therapy was planned for painful symptomatology remission., $3,11,12$

Chronic pain is a great burden to society, responsible for personal suffering, drug abuse, and a considerable financial problem due to treatment spending and lost productivity. ${ }^{10}$ A study from 2012 found that the annual cost of pain in the United States ranged from $\$ 560$ to $\$ 635$ billion dollars, being superior to the costs of heart disease ( $\$ 309$ billion), cancer ( $\$ 243$ billion), and diabetes ( $\$ 188$ billion). ${ }^{20}$ In this context, acupuncture can play an important role considering its efficacy in reducing pain, few occurrence of adverse effects, and relatively low cost. ${ }^{10}$

Acupuncture has been often used as a complementary treatment in pain management in TMD. ${ }^{3} \mathrm{~A}$ study comparing dry needling (DN) with methocarbamol (380 $\mathrm{mg}$ ) and paracetamol (300 $\mathrm{mg}$ ) combination drug therapy in TMD patients with pain on the lateral pterygoid muscle showed better results in the DN group. ${ }^{21}$ In a clinical trial involving occlusal splints, a group treated only with splint and another only with acupuncture had significant and similar results on pain reduction. ${ }^{2}$ In another study, the group treated with a combination of occlusal splint and laser acupuncture had faster and greater pain reduction than the group treated with occlusal splint and laser placebo. ${ }^{4}$

According to Western medicine, acupuncture is responsible for activating peripheral sensory nerves and the afferent nerve pathway in the spinal cord, liberating cortisol, enkephalins, endorphins, dynorphins, and endomorphins blocking the painful stimulus. ${ }^{10,16,22}$ In the reported case, the patient was in great pain before treatment (score 10 on VAS) and pain medications were not being sufficiently effective, reducing pain to a score of 6 on VAS according to the patient. Acupuncture treatment could eliminate patient's pain without the intake of any substance and the patient had no side effects and as was observed on the EMG test, muscular activity reduced both in $\mathrm{T} 1$ and $\mathrm{T} 2$ for most of the evaluated positions ( $\boldsymbol{- T a b l e ~} \mathbf{1}$ ).

Chronic pain is characterized by the International Association for the Study of Pain as the one which persists for more than 6 months. ${ }^{23}$ Pain perception during the first 6 months is considered more trustworthy because the patient can better comprehend the location and duration of it, where the discriminative system dominates the motivational/effective one, herewith the individual can not only just to locate but also isolate it. ${ }^{9,24,25}$ These information just highlight the 
Table 2 Selected acupoints description

\begin{tabular}{|c|c|c|c|}
\hline Body place & Point & Anatomical position & Indication \\
\hline \multirow[t]{6}{*}{ Facial } & Jiache (ST6) & $\begin{array}{l}\text { On the cheek, } 1 \text { cun superior to the } \\
\text { anterior angle and above the mandible, on } \\
\text { masseter muscle prominence }\end{array}$ & $\begin{array}{l}\text { Relax facial muscles, facilitate mandible } \\
\text { opening. Improves TMJ Qi }\end{array}$ \\
\hline & Xianguan (ST7) & $\begin{array}{l}\text { On the face, distally to the zygomatic bone, } \\
\text { in the center of the depression between } \\
\text { the mandible incisure and the inferior edge } \\
\text { of the zygomatic arch, anterior to the man- } \\
\text { dibular condylar process, on the posterior } \\
\text { margin of masseter muscle }\end{array}$ & $\begin{array}{l}\text { Improve ear and TMJ functions. Alleviate } \\
\text { spasms and pain }\end{array}$ \\
\hline & Tinggong (SI19) & $\begin{array}{l}\text { On the face, anatomical depression formed } \\
\text { when mouth opens, localized anterior to } \\
\text { the ear tragus, between the TMJ and the } \\
\text { tragus }\end{array}$ & $\begin{array}{l}\text { Relax the Shen, benefits the mind, } \\
\text { removes Qi and blood obstructions of } \\
\text { the vessels, alleviates pain, muscular } \\
\text { spasms, trismus, TMJ motor impairment, } \\
\text { and toothaches }\end{array}$ \\
\hline & Ermen (TE21) & $\begin{array}{l}\text { Superior part of the cavity before the } \\
\text { tragus and above the mandibular condyle } \\
\text { as mouth opens }\end{array}$ & $\begin{array}{l}\text { Toothaches and pains, TMJ arthritis, } \\
\text { headaches }\end{array}$ \\
\hline & Yintang & $\begin{array}{l}\text { Anterior middle line of the face, middle } \\
\text { point between the eyebrows }\end{array}$ & Extra point that eases the mind \\
\hline & Trigger points & $\begin{array}{l}\text { A palpable taut band anywhere on the } \\
\text { body }\end{array}$ & $\begin{array}{l}\text { Relaxation of the region and pain } \\
\text { reduction }\end{array}$ \\
\hline \multirow[t]{6}{*}{ Systemic } & Bai Hui (GV20) & $\begin{array}{l}\text { Middle of the skull, at the top of the head, } \\
\text { on the intersection of the body middle line } \\
\text { with the line from the vertical axis of both } \\
\text { ears }\end{array}$ & Stimulates the memory and the mind \\
\hline & Hegu (LI4) & $\begin{array}{l}\text { Radial face of the hand, between meta- } \\
\text { carpal bones I and II, closer to the second } \\
\text { one, approximately on its center, on the } \\
\text { muscular projection when adduction of the } \\
\text { thumb is performed }\end{array}$ & $\begin{array}{l}\text { Eases the mind, decreases pain and } \\
\text { inflammation, improves gastrointestinal } \\
\text { function and headaches }\end{array}$ \\
\hline & Houxi (SI3) & $\begin{array}{l}\text { On the ulnar margin on the hand, in the } \\
\text { depression proximal to the metacarpal } \\
\text { bone head V }\end{array}$ & $\begin{array}{l}\text { Alleviates pain and releases articular } \\
\text { movements }\end{array}$ \\
\hline & $\begin{array}{l}\text { Yanglingquan } \\
\text { (GB34) }\end{array}$ & $\begin{array}{l}\text { Superior third of the leg lateral face, on the } \\
\text { muscular depression anterior and inferior } \\
\text { to the fibular head }\end{array}$ & $\begin{array}{l}\text { Alleviates pain, decreases anger, and } \\
\text { relaxes the TMJ }\end{array}$ \\
\hline & Fengchi (GB20) & $\begin{array}{l}\text { Lateral and posterior to the neck, on the } \\
\text { bone depression between external occipital } \\
\text { tuberosity and mastoid process, at Fengfu } \\
\text { level, on the depression between the ster- } \\
\text { nocleidomastoid muscle and the superior } \\
\text { margin of the trapezius }\end{array}$ & $\begin{array}{l}\text { Relax the muscles and tendons, } \\
\text { improves articular functions }\end{array}$ \\
\hline & Zusanli (ST36) & $\begin{array}{l}\text { Anterolateral face of the leg, } 3 \text { cun directly } \\
\text { below the Dubi and } 1 \text { cun lateral to the } \\
\text { anterior margin of the tibia between the } \\
\text { muscles tibialis anterior and the extensor } \\
\text { digitorum }\end{array}$ & $\begin{array}{l}\text { Stomach tonification, alleviates pain and } \\
\text { inflammation, improves gastrointestinal } \\
\text { function and headaches }\end{array}$ \\
\hline
\end{tabular}

Source: Adapted from Han et al. ${ }^{16}$

fidelity of the painful symptomatology intensity related to the patient on this study, not only the initial but also after the treatment, in as much as they began approximately 2 months prior to therapy beginning, being still considered acute, allowing a better perception of the patient regarding the painful spots, which not only contributed to the diagnosis as for treatment application, especially on the painful points. And the importance of a quick intervention for painful symptomatology remission is indispensable for quality of life maintenance and effectiveness on the patient's routine.

\section{Conclusion}

Myofascial pain is one of the main symptoms of TMD and treatments must act on pain control. ${ }^{1-3,6,11}$ The patient's psychological and emotional status are risk factors for the 
uprising or aggravation of the painful symptomatology. 3.,4,11,19 In this case report, acupuncture was chosen before occlusal splint therapy because of its effectiveness on acting quickly on TMD painful symptoms as was observed by the absence of pain after acupuncture treatment since the first session, directly impacting the patient's well-being considering that pain was present for 2 months and medications were not working effectively anymore. However, acupuncture alone does not cure TMD due to its multifactorial etiology which often requires multidisciplinary approaches that not only treat painful symptomatology but also work to eliminate the causes.

\section{Funding}

This study was financed in part by the Coordenação de Aperfeiçoamento de Pessoal de Nível Superior - Brasil (CAPES) - Finance Code 001.

\section{Conflict of Interest}

None declared.

\section{References}

1 De Rossi SS, Greenberg MS, Liu F, Steinkeler A. Temporomandibular disorders: evaluation and management. Med Clin North Am 2014;98(6):1353-1384

2 Grillo CM, Canales GdeL, Wada RS, et al. Could acupuncture be useful in the treatment of temporomandibular dysfunction? J Acupunct Meridian Stud 2015;8(4):192-199

3 Fernandes AC, Duarte Moura DM, Da Silva LGD, De Almeida EO, Barbosa GAS. Acupuncture in temporomandibular disorder myofascial pain treatment: a systematic review. J Oral Facial Pain Headache 2017;31(3):225-232

4 Ferreira LA, de Oliveira RG, Guimarães JP, Carvalho AC, De Paula MV. Laser acupuncture in patients with temporomandibular dysfunction: a randomized controlled trial. Lasers Med Sci 2013;28(6):1549-1558

5 Sener S, Akgunlu F. Correlation between the condyle position and intra-extraarticular clinical findings of temporomandibular dysfunction. Eur J Dent 2011;5(3):354-360

6 Gauer RL, Semidey MJ. Diagnosis and treatment of temporomandibular disorders. Am Fam Physician 2015;91(6):378-386

7 Carvalho LSC, Nascimento OJM, Rodrigues LL, Matta APDC. Relationship between expanded disability status scale scores and the presence of temporomandibular disorders in patients with multiple sclerosis. Eur J Dent 2018;12(1):144-148

8 Kindler S, Samietz S, Houshmand M, et al. Depressive and anxiety symptoms as risk factors for temporomandibular joint pain: a prospective cohort study in the general population. J Pain 2012;13(12):1188-1197

9 Alrashdan MS, Alkhader M. Psychological factors in oral mucosal and orofacial pain conditions. Eur J Dent 2017;11(4): 548-552
10 Fan AY, Miller DW, Bolash B, et al. Acupuncture's role in solving the opioid epidemic: evidence, cost-effectiveness, and care availability for acupuncture as a primary, non-pharmacologic method for pain relief and management-white paper 2017. J Integr Med 2017;15(6):411-425

11 Branco CA, Fonseca RB, Borges RF, Venezian GC, Magri LV, Mazzetto MO. Perception of the signs and symptoms of temporomandibular disorder in females by using the ProTMDMulti protocol and the visual analog scale before and after acupuncture treatment. Cranio 2016;34(2):118-123

12 Itoh K, Asai S, Ohyabu H, Imai K, Kitakoji H. Effects of trigger point acupuncture treatment on temporomandibular disorders: a preliminary randomized clinical trial. J Acupunct Meridian Stud 2012;5(2):57-62

13 Dworkin SF, LeResche L. Research diagnostic criteria for temporomandibular disorders: review, criteria, examinations and specifications, critique. J Craniomandib Disord 1992;6(4):301-355

14 Politti F, Casellato C, Kalytczak MM, Garcia MB, BiasottoGonzalez DA. Characteristics of EMG frequency bands in temporomandibullar disorders patients. J Electromyogr Kinesiol 2016;31:119-125

15 Sacco IC, Gomes AA, Otuzi ME, Pripas D, Onodera AN. A method for better positioning bipolar electrodes for lower limb EMG recordings during dynamic contractions. J Neurosci Methods 2009;180(1):133-137

16 Han JS. Acupuncture analgesia: areas of consensus and controversy. Pain 2011;152(3, Suppl):S41-S48

17 Fricton J. Myogenous temporomandibular disorders: diagnostic and management considerations. Dent Clin North Am 2007;51(1):61-83

18 Okeson JP, de Leeuw R. Differential diagnosis of temporomandibular disorders and other orofacial pain disorders. Dent Clin North Am 2011;55(1):105-120

19 Rai S, Ranjan V, Misra D, Panjwani S. Management of myofascial pain by therapeutic ultrasound and transcutaneous electrical nerve stimulation: a comparative study. Eur J Dent 2016;10(1):46-53

20 Gaskin DJ, Richard P. The economic costs of pain in the United States. J Pain 2012;13(8):715-724

21 Gonzalez-Perez LM, Infante-Cossio P, Granados-Nunez M, Urresti-Lopez FJ, Lopez-Martos R, Ruiz-Canela-Mendez P. Deep dry needling of trigger points located in the lateral pterygoid muscle: efficacy and safety of treatment for management of myofascial pain and temporomandibular dysfunction. Med Oral Patol Oral Cir Bucal 2015;20(3):e326-e333

22 Maciocia G, The Foundations of Chinese Medicine, 3rd ed. Oxford: Churchill Livingstone; 2015

23 Merrill RL. Central mechanisms of orofacial pain. Dent Clin North Am 2007;51(1):45-59

24 Auvenshine RC. Temporomandibular disorders: associated features. Dent Clin North Am 2007;51(1):105-127

25 Auvenshine RC. Acute vs. chronic pain. Tex Dent J 2000;117(7): $14-20$ 